\title{
Detection of prokaryotic promoters from the genomic distribution of hexanucleotide pairs Pierre-Étienne Jacques ${ }^{1,2}$, Sébastien Rodrigue ${ }^{1}$, Luc Gaudreau ${ }^{1}$, Jean Goulet ${ }^{2}$ and Ryszard Brzezinski*1,3
}

\author{
Address: ${ }^{1}$ Département de biologie, Université de Sherbrooke, Sherbrooke, Québec, Canada, ${ }^{2}$ Département d'informatique, Université de \\ Sherbrooke, Sherbrooke, Québec, Canada and ${ }^{3}$ Centre d'étude et de valorisation de la diversité microbienne, Université de Sherbrooke, \\ Sherbrooke, Québec, Canada \\ Email: Pierre-Étienne Jacques - Pierre-Etienne.Jacques@USherbrooke.ca; Sébastien Rodrigue - Sebastien.Rodrigue@USherbrooke.ca; \\ Luc Gaudreau - Luc.Gaudreau@USherbrooke.ca; Jean Goulet - Jean.Goulet@USherbrooke.ca; \\ Ryszard Brzezinski* - Ryszard.Brzezinski@USherbrooke.ca \\ * Corresponding author
}

Published: 02 October 2006

BMC Bioinformatics 2006, 7:423 doi:10.1186/147/-2105-7-423
Received: 05 May 2006

Accepted: 02 October 2006

This article is available from: http://www.biomedcentral.com/I47I-2/05/7/423

(C) 2006 Jacques et al; licensee BioMed Central Ltd.

This is an Open Access article distributed under the terms of the Creative Commons Attribution License (http://creativecommons.org/licenses/by/2.0), which permits unrestricted use, distribution, and reproduction in any medium, provided the original work is properly cited.

\begin{abstract}
Background: In bacteria, sigma factors and other transcriptional regulatory proteins recognize DNA patterns upstream of their target genes and interact with RNA polymerase to control transcription. As a consequence of evolution, DNA sequences recognized by transcription factors are thought to be enriched in intergenic regions (IRs) and depleted from coding regions of prokaryotic genomes.

Results: In this work, we report that genomic distribution of transcription factors binding sites is biased towards IRs, and that this bias is conserved amongst bacterial species. We further take advantage of this observation to develop an algorithm that can efficiently identify promoter boxes by a distribution-dependent approach rather than a direct sequence comparison approach. This strategy, which can easily be combined with other methodologies, allowed the identification of promoter sequences in ten species and can be used with any annotated bacterial genome, with results that rival with current methodologies. Experimental validations of predicted promoters also support our approach.
\end{abstract}

Conclusion: Considering that complete genomic sequences of over 1000 bacteria will soon be available and that little transcriptional information is available for most of them, our algorithm constitutes a promising tool for the prediction of promoter sequences. Importantly, our methodology could also be adapted to identify DNA sequences recognized by other regulatory proteins.

\section{Background}

Adaptation is essential to the survival of any biological organism and requires appropriate transcriptional regulation to modulate gene expression profiles. In prokaryotes, RNA polymerase (RNAP) is responsible for the transcrip- tion of all genes. However, promoter recognition is effected by an interchangeable sigma $(\sigma)$ factor that associates to RNAP, directs the newly formed holoenzyme to a promoter and contributes to transcription initiation [1]. Transcription levels can be further modified by additional 
regulators (activators and repressors) that affect the recruitment or the activity of RNAP holoenzymes at various promoters [2]. A common feature of $\sigma$ factors and transcriptional regulators is their ability to recognize specific DNA patterns in order to modulate gene expression. It is presumed that, as a result of evolutionary pressure, these regulatory sequences were selected upstream of some genes or operons and excluded from the rest of the genome.

Bacterial genomes usually encode many $\sigma$ factors. Of these, the principal $\sigma$ factor would be responsible for the expression of housekeeping function genes. The remaining $\sigma$ factors are thought to direct the expression of genes required for specialized functions such as stress responses or sporulation [1]. $\sigma$ factors can also be classified according to their structural homology to either $\sigma^{70}$ or $\sigma^{54}$ of Escherichia coli. $\sigma^{70}$-related factors, which constitute the vast majority of known $\sigma$ factors, are composed of two major DNA-binding domains capable of sensing a certain spacing range when associated to RNAP $[3,4]$. These $\sigma$ factors usually recognize two DNA boxes (herein referred as the promoter) of approximately six base pairs (bp) located roughly at 10 and 35 bp upstream of the transcription start site (TSS). Spacing between these two boxes generally ranges from 16 to $20 \mathrm{bp}$ [5]. $\sigma$ factors similar to $\sigma^{54}$ also recognize two DNA boxes in the promoter region. However these elements are located approximately 12 and 24 bp upstream of the TSS. Other major differences between $\sigma^{54}$ and $\sigma^{70}$ family members are the ability of $\sigma^{54}$ to bind DNA in absence of RNAP and the requirement of an isomerization step by an activator to render $\sigma^{54}$-containing holoenzymes processive.

$\sigma$ factors can tolerate a variety of mismatches from their consensus sequence. For example, a typical E. coli $\sigma^{70}$ promoter sequence contains two mismatches within both the -35 and -10 hexanucleotide elements [6]. However, there is generally a direct relationship between promoter strength and the similarity to the corresponding consensus sequence [7]. Variations over three orders of magnitude have been reported in $\sigma^{70}$-dependent promoter strength in E. coli [8]. In some cases, an extended -10 promoter box may be observed and may substitute for the absence of a clear -35 element. Extended -10 promoter boxes were reported to be present in $20 \%$ of promoter sequences in E. coli [6] and 45\% in Bacillus subtilis [5].

A variety of techniques have been used to identify TSS and to characterize $\sigma$ factor-DNA interactions. However, the formal identification of promoters by molecular methods can be tedious and is currently not amenable to genomewide applications. Consequently, it is important to develop algorithms that can rapidly and accurately evaluate the presence of promoters, without the need for exten- sive biochemical studies. Current algorithms for promoter detection, typically developed for a specific bacterium, exploit different characteristics of promoter sequences. Some approaches are based on sequence representation or statistical overrepresentation. Other methodologies have also been described for the detection of DNA motifs in sets of regulatory sequences [9-12] or by comparing the upstream regions of orthologous genes from different species [13-17]. A method based on the weaker stability of the DNA double-helix in promoter regions was also recently used to identify promoter regions [18]. However, most of these procedures are not suitable for the identification of precise prokaryotic promoters because of the inherent variability in promoter sequences and because they do not allow variable spacers between two DNA motifs.

Sequence representation strategies designed for promoter identification are usually based on a prior knowledge of some characterized sequences. These algorithms are thus trained to recognize sequences that are similar to a previously defined representation of a promoter. This approach was first used by Galas et al. [19]. As reported by Stormo, numerous false positives (FP) are however obtained with this strategy [20]. For example, allowing two mismatches in the $\sigma^{70}$ consensus -10 hexanucleotide produces roughly one hit per 30 nucleotides (nt) in the complete genome of $E$. coli. A more accurate representation of DNA-binding motifs consists of position-specific weight matrices (PSWM) [21], and online tools such as Virtual Footprint [22] are available to facilitate their analysis in the context of bacterial gene expression. Nonetheless, searching for full E. coli $\sigma^{70}$ consensus promoter sequences using more flexible mismatch restrictions offered by PSWM also generates a vast amount of hits [23]. More recently, Huerta and Collado-Vides used a PSWM-derived methodology and detected approximately 15 putative promoters/100 nt in IRs [24]

By adding several constraints such as grouping sequences and filtering with the distance from the start codon, they achieved a sensitivity of $86 \%$ with an average of 1.88 putative promoters/100 nt. Several groups have also used general neural networks but no significant improvements have been achieved over the PSWM [25]. Hidden Markov Models (HMM) have also been trained to identify promoter sequences recognized by the principal $\sigma$ factor in $B$. subtilis [26] and Campylobacter jejuni [27]. A learning approach based on a Support Vector Machine (SVM) employing a variant of the mismatch string kernel was also recently described [28]. Importantly, all above-mentioned approaches depend on a previously established or trained description of promoters, and were not designed to function with organisms for which promoter information is insufficient. 
Statistical overrepresentation approaches can identify short DNA sequences that are present more frequently in a subset of sequences than what would be expected by chance according to the background distribution. Using such a procedure, Vanet $e t$ al. have proposed a description of the promoter sequences recognized by the principal $\sigma$ factor of Helicobacter pylori from different sets of IRs [29]. More recently, the MITRA algorithm, which also evaluates the spacing between promoter boxes and the positional bias from the start codon, was applied to 20 bacterial genomes. Four of these genomes generated statistically strong signals possibly corresponding to principal $\sigma$ factor-dependent promoter sequences, including the ones from $H$. pylori and B. subtilis [30]. Using a different approach, the principal $\sigma$ factor consensus sequence was identified among over-represented motifs in B. subtilis, although the methodology was not designed especially for that purpose [31]. The latter study was based on the method of Li et al., designed to identify regulatory protein binding sites in E. coli [32]. It has been noticed that the $E$. coli $\sigma^{70}$ consensus sequence was not identified by this or other approaches, a failure that was attributed to the greater variability of promoter sequences within this organism [32]. A similar method was also unable to distinguish a motif related to the principal $\sigma$ factor promoters in the complete genome of Streptomyces coelicolor [33]. In general, although some statistical approaches had limited success, these methods do not seem appropriate in their current form for the identification of promoter sequences in a variety of organisms.

In this paper, we describe a novel approach based on matrices representing the genomic distribution of hexanucleotide pairs, and designed to predict precise promoter sequences using any annotated prokaryotic genome. This approach can be applied to organisms for which almost no transcriptional data is available, without the need for extensive biochemical characterization. The strategy is based on the observation that, although promoter sequences can vary for every $\sigma$ factor and according to the GC content of each genome [34], promoters are over-represented in IRs relative to the whole genome. Since this bias appears to be conserved throughout evolution, the characteristic distribution of promoter sequences is thus used to identify promoters in a variety of prokaryotic organisms. Briefly, a score is calculated based on the similarity between a matrix representing the genomic distribution of most promoter sequences reported in the literature and a matrix representing the genomic distribution of a putative promoter sequence. A Z-score is next calculated according to the background. To assess the validity of our method, over 680 characterized promoter sequences from ten genomes were gathered from databases and from the literature, and tested using various sta- tistical indicators. Experimental validations of promoter prediction also supported our approach.

\section{Results \\ Genomic distribution of regulatory sequences is biased and conserved}

Transcription initiation is an important step in the regulation of most bacterial cell processes [35,36]. For this reason, it is thought that transcription factor binding sites have been evolutionary selected in some IRs, and generally excluded from the rest of prokaryotic genomes in order to avoid aberrant gene expression. Since regulatory sequences can often tolerate a variety of mismatches with respect to the corresponding DNA motif consensus sequence and still be efficiently bound by their cognate DNA binding protein, these degenerated sequences are also believed to be subjected to a similar selective pressure. Therefore, the genomic distribution of transcription factor consensus DNA binding sequences and close derivatives is expected to be biased towards IRs possibly involved in transcription initiation relative to the whole genome. To test this latter hypothesis, we have analyzed the genomic distribution of consensus sequences representing, respectively, the recognized sequences of a principal $\sigma$ factor, an alternative $\sigma$ factor and a transcriptional regulator of three bacterial species (Figure 1 and data not shown). The global distribution of these DNA sequences is reported in "distribution matrices", which consists of tables of dimension $4 \times 4$ where lines and columns correspond respectively to the number of mismatches in the proximal and distal boxes of a particular DNA sequence. For every matrix cells, the proportion of hits in putative promoter-containing IRs $(\mathrm{P})$ relative to the whole genome (G) was calculated, with respect to a defined spacing range between the two hexanucleotide boxes forming the evaluated sequences. Light colored cells correspond to low P/G ratios while darker cells represent higher frequencies. For example, the genome-derived distribution matrix of the $E$. coli $\sigma^{70}$ consensus promoter sequence shows that $65 \%$ of genome sequences bearing one mismatch in the -35 element (distal box) but a perfect -10 (proximal) box are localized in $\mathrm{P}$ regions relative to the whole genome. At three mismatches in each box $(50 \%$ degeneracy of the analyzed sequence), about $16 \%$ of the hits are found in $\mathrm{P}$ regions, which roughly corresponds to the proportion of IRs in the genome. Such sequences are thus considered randomly distributed in the genome. Results presented in Figure 1 thus demonstrate that transcription factor consensus DNA binding sites and related mismatch(es)-containing derivatives tend to be preferentially localized inside IRs. Moreover, the genomic distribution of these sequences shows related patterns in all tested bacterial species (Figure 1). 

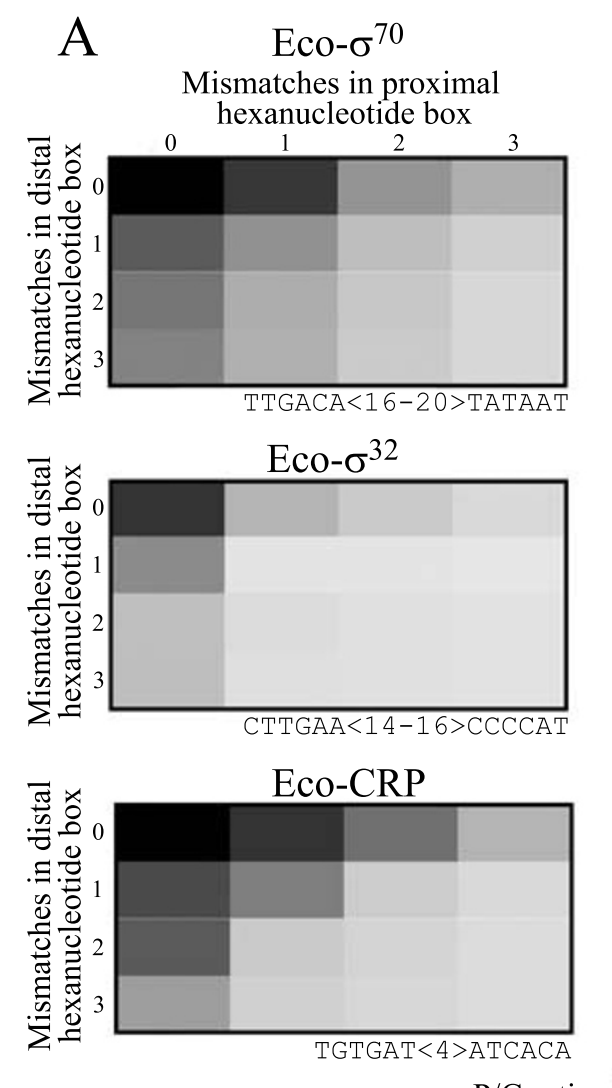

$\mathrm{P} / \mathrm{G}$ ratios: $0 \%$
B
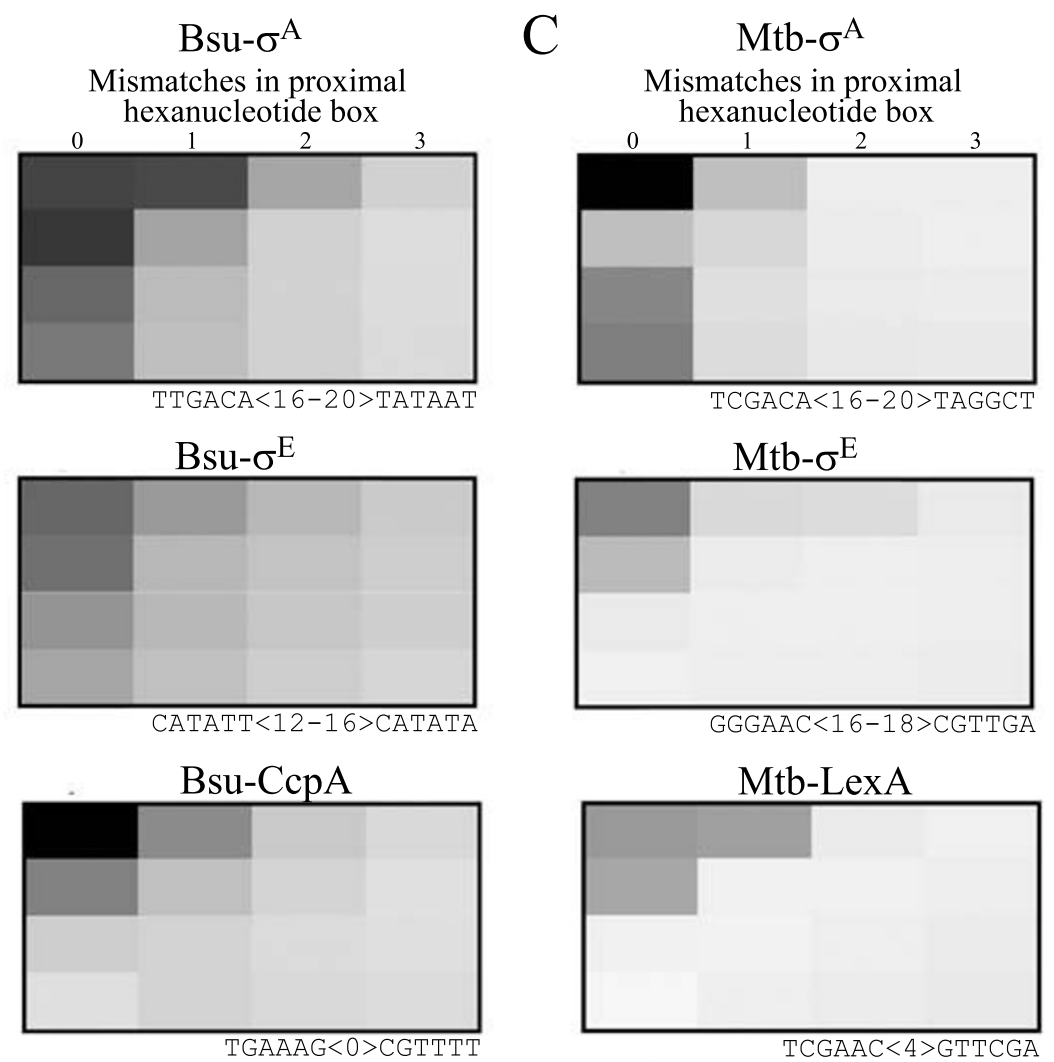

TGAAAG $<0>$ CGTTTT

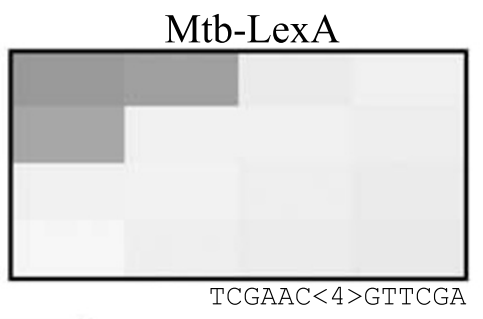

$100 \%$

\section{Figure I}

Genome-derived distribution matrices generated for consensus sequences from transcription factors of three different organisms. (A) E. coli, (B) B. subtilis, and (C) M. tuberculosis. The name of the transcription factor is identified above each matrix. The mismatch number of each cell is indicated on both sides of matrices. The analyzed consensus sequence is shown under each matrix, along with the allowed spacing range. The first row corresponds to principal $\sigma$ factors, the second row to alternative $\sigma$ factors, and the third row to transcriptional regulators.

\section{Principal $\sigma$ factor promoter sequences}

Because principal $\sigma$ factor promoter sequences are readily available and relatively abundant in many bacterial species, we decided to further explore their genomic distribution. Figure 2 (upper and middle rows) shows genomederived distribution matrices of experimentally identified promoter sequences from E. coli, B. subtilis and M. tuberculosis. As expected, these matrices contain patterns similar to those observed with the corresponding principal $\sigma$ factor consensus sequences (Figure 1). Importantly, the genomic distribution of sequences from the coding region of the $r p o B$ genes from the same bacterial species did not show any enrichment inside of $\mathrm{P}$ regions (Figure 2, last row and data not shown). These results suggest that transcription factor DNA binding sites from various bacterial species have a genomic distribution significantly different from that of non-regulatory sequences.

\section{Synthetic matrices}

From these observations, we hypothesized that a particular genome-derived distribution matrix may appropriately represent several promoters. Hence, it could be possible to calculate a score reflecting the similarity between the distribution matrix of a typical promoter and the genomederived distribution matrix of any hexanucleotide pair from the same organism. A high score would indicate a strong probability that the tested sequence is also a promoter. However, the best reference matrix should not necessarily be obtained from an existing promoter sequence. In fact, an interpolated matrix could indeed offer more flexibility and be much more effective. We therefore decided to synthetically generate distribution matrices according to the values observed in each cell of the genome-derived distribution matrices for all available experimentally identified principal $\sigma$ factor-dependent 

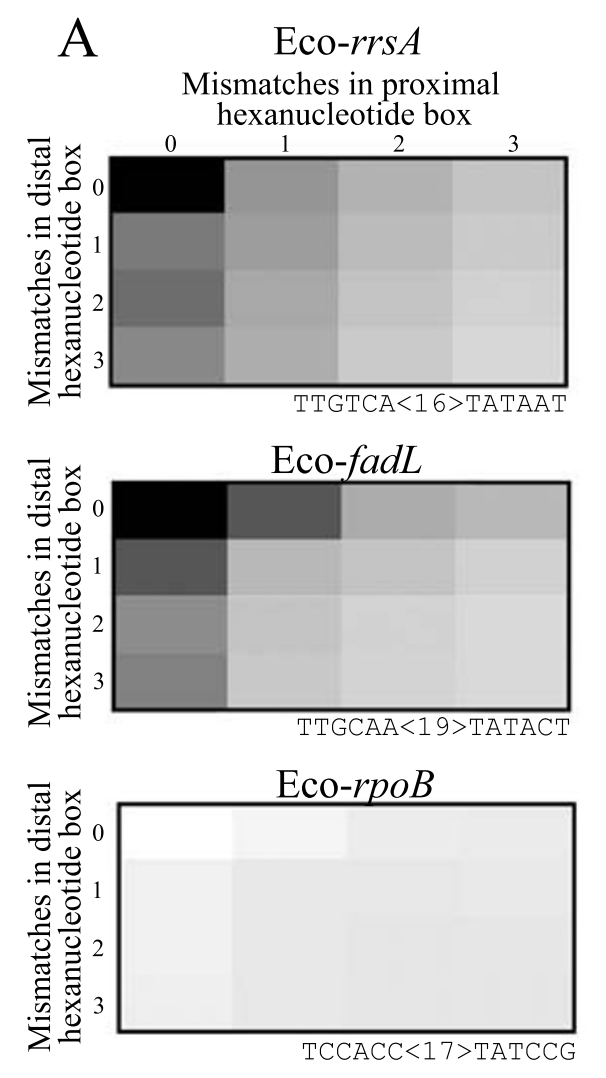

$\mathrm{P} / \mathrm{G}$ ratios: $0 \%$
B
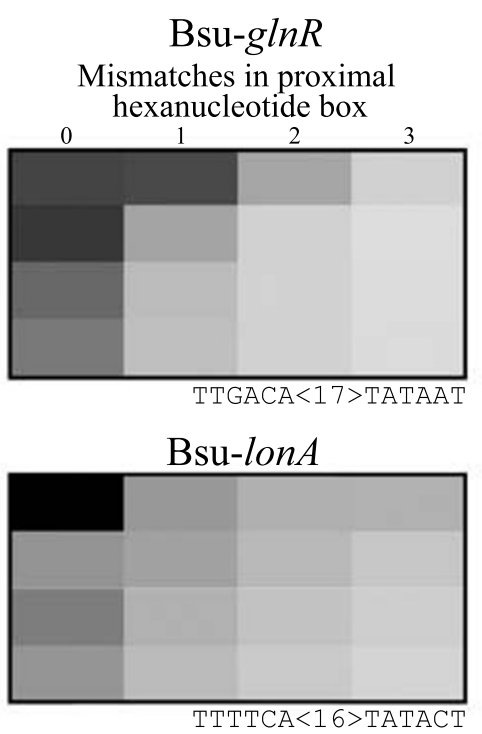

Bsu-rpo $B$
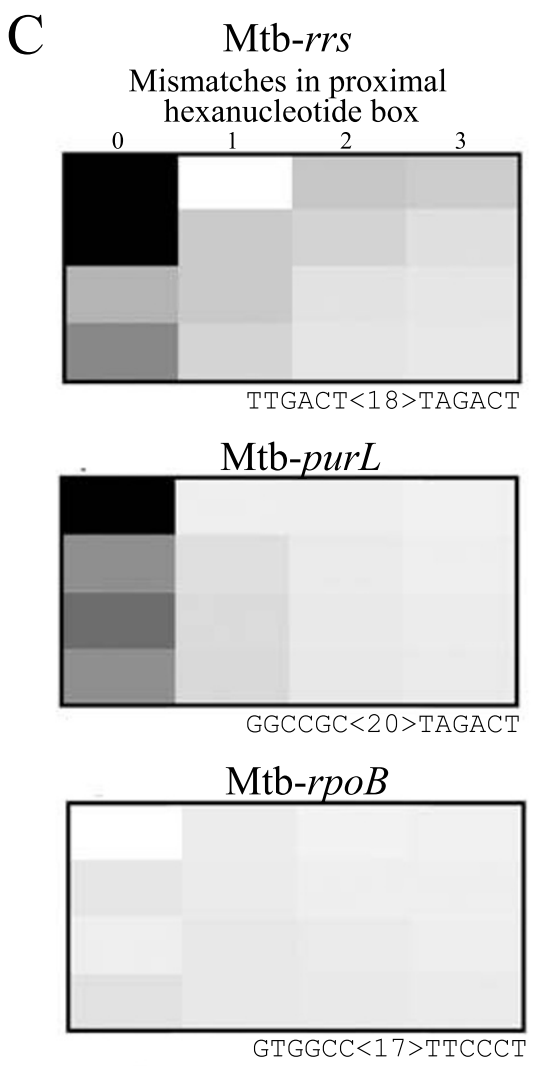

$100 \%$

Figure 2

Specific examples of genome-derived distribution matrices generated for characterized principal $\sigma$ factordependent promoter sequences and non-regulatory sequences from three different organisms. (A) $E$. coli, (B) $B$. subtilis, and (C) M. tuberculosis. Gene names and experimentally identified promoter sequences, as reported in the literature, are indicated above each matrix. The first row corresponds to characterized promoter sequences closely resembling to the proposed consensus. The second row presents experimentally identified promoters containing more mismatches relative to the proposed consensus. The last row shows distribution matrices of hexanucleotide pairs with approximately 3 mismatches position per box, which were extracted from the middle of the rpoB coding sequence (bona fide non-promoter sequences).

promoter sequences (see Additional file 1: A schematic description of the procedures used in this work). A range of ratios was next determined for each cell, resulting in over 248 million different synthetic matrices (see Additional file 2: Detailed information on the generation of synthetic matrices). Synthetic matrices are thus not affiliated to any hexanucleotide pairs, but are rather produced from the genome-derived distribution matrices of experimentally identified promoters. Moreover, it could be possible to identify synthetic matrices suitable to detect promoter sequences for a specific organism ("organismspecialized matrix"), and perhaps for all bacteria ("general matrix").

\section{Using distribution matrices to detect promoters}

To measure the ability of synthetic matrices to correctly identify promoter sequences, we tested them on 684 characterized principal $\sigma$ factor dependent promoter sequences from ten bacterial species (Table 1). Complete IRs containing these promoters were extracted from their respective genomes along with $30 \mathrm{nt}$ on each side. These "enlarged IRs" were next scanned using two hexanucleotide windows with respect to the allowed spacing ranges. For each hexanucleotide pair, a genome-derived distribution matrix was generated and used to calculate a score based on its similarity to the synthetic matrix under evaluation (see Additional file 3: Detailed example of score 
Table I: Analysis of characterized promoter sequences in ten bacterial genomes.

\begin{tabular}{|c|c|c|c|c|c|c|c|c|c|c|}
\hline \multirow[b]{2}{*}{ Organism } & \multirow[b]{2}{*}{ GC } & \multirow[b]{2}{*}{ Prom. } & \multirow[b]{2}{*}{ IRs } & \multirow[b]{2}{*}{$\mathbf{N t}$} & \multirow[b]{2}{*}{ Spacing } & \multicolumn{3}{|c|}{ Organism-specialized matrices } & \multicolumn{2}{|c|}{ General matrix } \\
\hline & & & & & & Sensitivity & FP/I00 nt & Name & Sensitivity & FP/I00 nt \\
\hline E. coli & $50.8 \%$ & 377 & 335 & 117238 & $16-20$ & $42.4 \%$ & 1.13 & 229794169 & $31.0 \%$ & 1.09 \\
\hline B. subtilis & $43.5 \%$ & 148 & 142 & 43446 & $16-20$ & $56.8 \%$ & 0.99 & 113362653 & $50.0 \%$ & 0.93 \\
\hline C. glutamicum & $53.8 \%$ & 34 & 33 & 13572 & $16-20$ & $29.4 \%$ & 1.36 & 223574489 & $14.7 \%$ & 1.36 \\
\hline M. pneumoniae & $40.0 \%$ & 30 & 27 & 7662 & $15-19$ & $43.3 \%$ & 1.08 & 235984178 & $30.0 \%$ & 1.07 \\
\hline M. tuberculosis & $65.6 \%$ & 28 & 25 & 7812 & $16-20$ & $57.1 \%$ & 0.74 & 223574908 & $50.0 \%$ & 0.84 \\
\hline S. coelicolor & $72.1 \%$ & 17 & 17 & 5756 & $16-20$ & $58.8 \%$ & 1.42 & 248361134 & $47.1 \%$ & 1.27 \\
\hline H. pylori & $38.9 \%$ & 17 & 16 & 4725 & $19-23$ & $47.1 \%$ & 0.53 & 91939249 & $35.3 \%$ & 0.70 \\
\hline C. jejuni & $30.5 \%$ & 14 & 14 & 2872 & $16-20$ & $42.9 \%$ & 0.84 & 107109675 & $35.7 \%$ & 0.77 \\
\hline B. japonicum & $64.1 \%$ & II & II & 4229 & $16-20$ & $90.9 \%$ & 0.73 & 192892765 & $90.9 \%$ & 0.97 \\
\hline S. aureus & $32.9 \%$ & 8 & 5 & 2206 & $16-20$ & $37.5 \%$ & 0.59 & 24624174 & $37.5 \%$ & 1.13 \\
\hline
\end{tabular}

For each organism, the GC content, the number of gathered characterized promoter sequences, the number of distinct IRs containing these promoters, the total number of nt in these IRs, and the allowed spacing between hexanucleotide pairs are indicated. The sensitivity and FP rate obtained with the best synthetic matrix (organism-specialized) and the general matrix are also shown.

calculation). Different metrics have been tested and the selected one provided the best outcome (data not shown). Since the -10 box has a more important role in transcription [37], the mean of scores sharing the same -10 box was calculated. This latter averaged score had to be greater than the threshold to consider the corresponding sequence as a candidate promoter. Furthermore, since the distance between the TSS and the proximal hexanucleotide box can slightly vary (which may cause the experimental identification of -10 boxes to be inaccurate), signals located at $\pm 4 \mathrm{nt}$ of an experimentally identified proximal hexanucleotide box were considered as true positives (TP, see Methods). Indicators such as sensitivity, specificity, precision, and performance were calculated for each synthetic matrix [38]. We chose to present the FP rate per $100 \mathrm{nt}$ rather than the precision indicator to facilitate comparison since the precision indicator does not take into account the length of analyzed regions (Table 1 ). In contrast with other studies [24,39], the analyzed regions were not limited to a maximum length, longer regions producing more FPs.

"Organism-specialized" synthetic matrices (selected on the basis of the performance and sensitivity indicators) gave interesting results for each tested organism (Table 1). For instance, the synthetic matrix \#113362653 identified almost $60 \%$ of promoters among the set of 148 characterized promoter sequences from B. subtilis with approximately one $\mathrm{FP} / 100 \mathrm{nt}$. Overall, the sensitivity of the best matrix for each organism ranges from $29.4 \%$ to $90.9 \%$ with 0.53 to $1.42 \mathrm{FP} / 100 \mathrm{nt}$ (Table 1 ). Amongst the FP, some could be uncharacterized real promoter sequences. Performance, precision and specificity indicators ranged respectively between 4.6-23.8\%, 5.2\%-24.4\% and 98.699.5\% (data not shown). Cross-validation tests were also conducted with E. coli and B. subtilis promoter datasets and matrices very similar to the organism-specialized syn- thetic matrices were identified. Moreover, the sensitivity and FP rate of these cross-validation matrices were comparable to the organism-specialized matrices obtained using complete datasets, demonstrating the robustness of the approach and suggesting that the various organism-specialized matrices are appropriate (see Additional file 4: Three fold cross-validation results).

Interestingly, several synthetic matrices identified a significant fraction of promoter sequences in all tested genomes. We thus undertook to find a matrix that would be the best compromise for promoter detection in all bacteria. This was achieved by summing up the relative performance indicator of each matrix on all genomes. The best "general" synthetic matrix (\#45012859) (Figure 3A and Additional file 3) usually showed a slight decrease in sensitivity relative to organism-specialized synthetic matrices (Table 1). Nonetheless, the sensitivity of this general synthetic matrix ranged from $14.7 \%$ to $90.9 \%$ with 0.70 to $1.36 \mathrm{FP} / 100 \mathrm{nt}$ (Table 1 ), which is comparable to other approaches for an equivalent FP rate (see Additional file 5: Comparison with other bacterial promoter prediction approaches). Furthermore, we have replaced our scoring function by a function based on PSWM. The results obtained with $E$. coli and B. subtilis normalized PSWM promoter datasets, showed a significantly decreased sensitivity and a lower FP rate in our design, suggesting that the genomic distribution of promoter sequences is more flexible, and hence more portable between species than current promoter sequence models (see Additional file 6: Comparison with normalized PSWM scoring function).

The scores of all possible hexanucleotide pairs for a specific enlarged IR can be presented in a graph. As an example, Figure 3 shows graphs obtained using the enlarged IRs containing the characterized promoter sequences pre- 
A

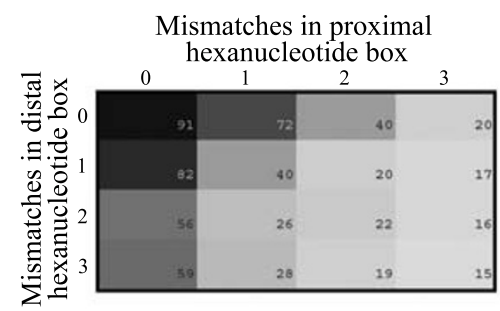

B
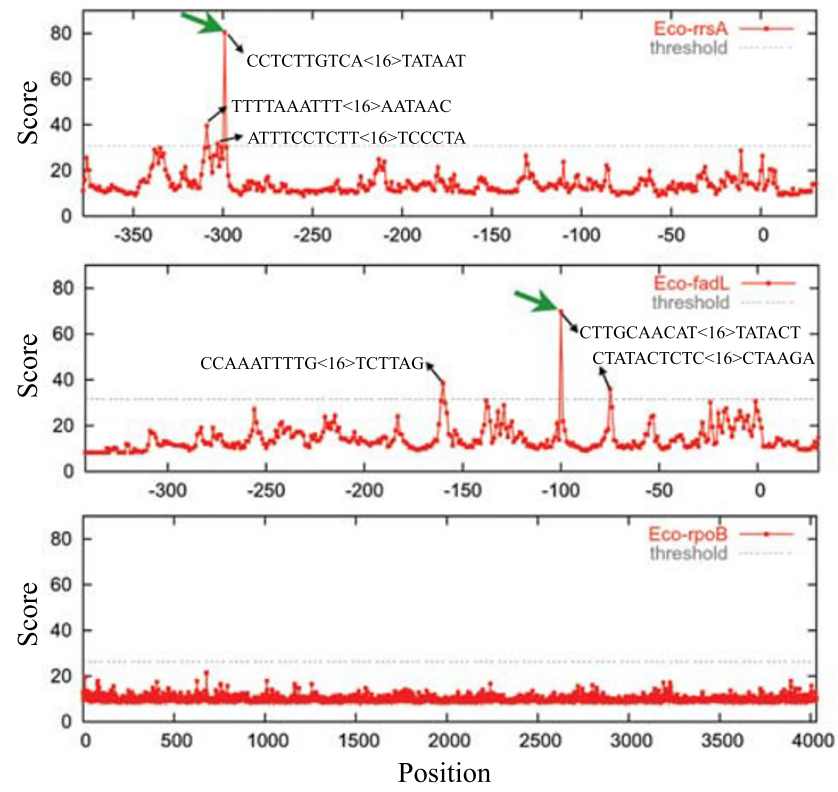

$\mathrm{C}$
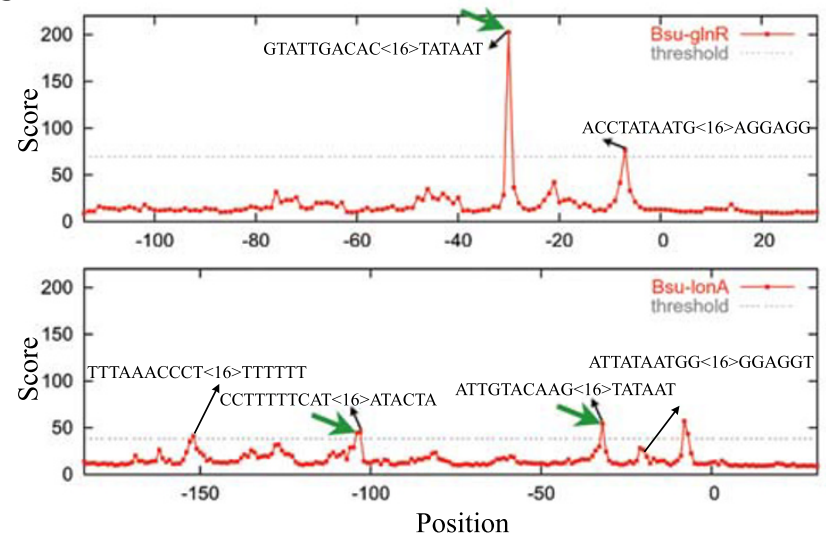

$\mathrm{D}$
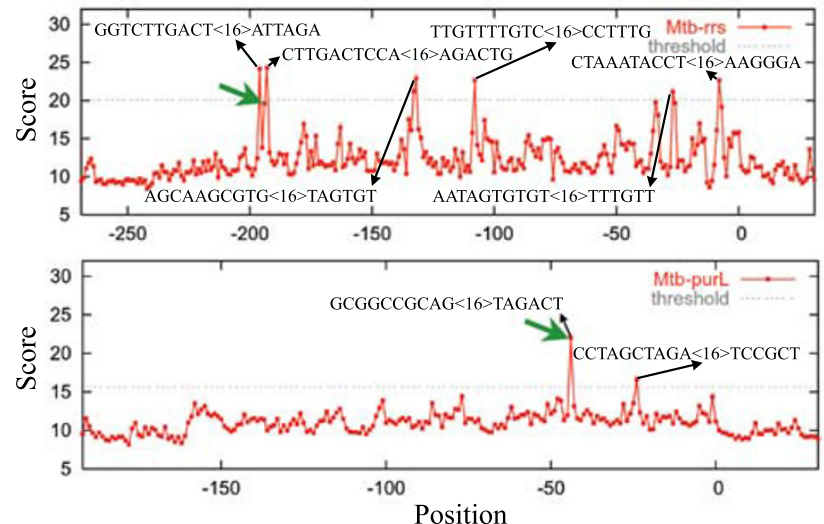

Figure 3

Graphs of enlarged IRs containing characterized promoter sequences presented in Figure 2. The green arrow represents the characterized promoter sequence. The start codon of the gene of interest is located at " 0 " on the $X$-axis of the enlarged IR. The $Y$-axis coordinate shows the calculated score. The threshold of each region is shown (dashed grey line). The sequence of all candidate promoters above the threshold is shown ( 5 merged overlapping - 35 boxes from the different allowed spacings along with the shared - I0 box). (A) The general synthetic matrix (\#450I2859) used to calculate the scores presented in the graphs. The name of the gene located downstream of the selected enlarged IR is indicated in each graph. $E$. coli (B), $B$. subtilis (C) and M. tuberculosis (D). Scores obtained for the full E. coli rpoB coding sequence were also plotted.

sented in Figure 2 for E. coli (Figure 3B), B. subtilis (Figure 3C) and M. tuberculosis (Figure 3D). The rpoB scanning is also presented for E. coli since identical results were obtained using the $\mathrm{B}$. subtilis and $\mathrm{M}$. tuberculosis sequences (Figure 3 and data not shown). Interestingly, Figure 3C (Bsu-lonA) shows one of many examples where a -10 promoter box (consensus TATAAT) is coupled to a ribosome binding site (RBS, consensus AGGAGG).

To assess the significance of our results, the analyses were repeated on two types of shuffled genome sequences. While retaining all the original genome information, the shuffling destroys its structure, which is used by our methodology to identify promoter sequences. The first shuffling procedure was accomplished by repositioning mononucleotides one region (gene or IR) at a time, thus keeping the AT bias intact in each IR [40]. The second shuffling was performed independently of gene annotations, thus dispersing the GC content uniformly through a genome. We surmised that most regulatory sequences, and by extension their genomic distribution, would be affected differently by these procedures. The overall sensitivity obtained with shuffled genomes should thus be decreased. Indeed, genome-derived distribution matrices were completely different if calculated from the real genome or from shuffled genomes (data not shown). As expected, the second shuffling was much more detrimental to the observed sensitivity. For instance, the calculated sensitivity with the general synthetic matrix dropped respectively from $31 \%$ (intact genome) to $14 \%$ and $0.6 \%$ 
(shuffled genomes) for E. coli, and from 50\% to, respectively $17 \%$ and $0.5 \%$ for $B$. subtilis. The same trend has been observed for other genomes (data not shown). Shuffling performed with longer nucleotides blocks than mononucleotide gave intermediate results (data not shown).

\section{Coupling sequence information to genome distribution}

For some bacteria, consensus promoter sequences representing the sequences recognized by principal $\sigma$ factors are well characterized. For example, the consensus promoter sequence for $B$. subtilis and E. coli is TTGACA < $>$ TATAAT [5]. We have thus examined the possibility of decreasing the occurrence of FPs by introducing a very simple sequence-dependent filter. Approximately half FPs were eliminated by allowing up to three mismatches in the -35 (distal) box and up to two mismatches in the -10 (proximal) box (the latter being more conserved in these organisms). For instance, a sensitivity of $49 \%$ with 0.50 $\mathrm{FP} / 100 \mathrm{nt}$ for the specialized matrix and $44 \%$ with 0.44 $\mathrm{FP} / 100 \mathrm{nt}$ for the general synthetic matrix was obtained for B. subtilis. Since fewer FPs were observed, the threshold could be lowered, which can improve the sensitivity of the algorithm. For example, the reduction of the IR specific threshold (see Methods) from three to two standard deviations upon sequence filtering gave a sensitivity of $58 \%$ with $0.80 \mathrm{FP} / 100 \mathrm{nt}$ in $B$. subtilis using the general synthetic matrix. Figure 4 illustrates how the application of a filter may allow the identification of an otherwise missed promoter.

\section{Prediction and biochemical validation of promoters}

In addition to the IRs used to evaluate synthetic matrices, we have applied our promoter detection algorithm on the remaining IRs from the ten genomes mentioned in Table 1. Some predicted promoters from B. subtilis, E. coli and M. tuberculosis were chosen for further investigation using a biochemical approach. RNA was extracted from exponentially growing cultures and TSS were determined by primer extension analyses. The seven candidate promoters for which a transcription signal was observed matched the predicted sequence (Table 2 ). The distance between the observed TSS and the potential -10 box was in all cases either 5 or $6 \mathrm{nt}$. These results demonstrate that our algorithm represent a valuable tool for the identification of prokaryotic promoter sequences. Complete prediction lists for these three organisms, and others, are available upon request.

\section{Discussion}

In this work, we present evidences suggesting that regulatory sequences and their close derivatives have a biased distribution pattern for IRs that may support transcription initiation. Furthermore, our data support the idea that the preferential location of regulatory sequences is shared
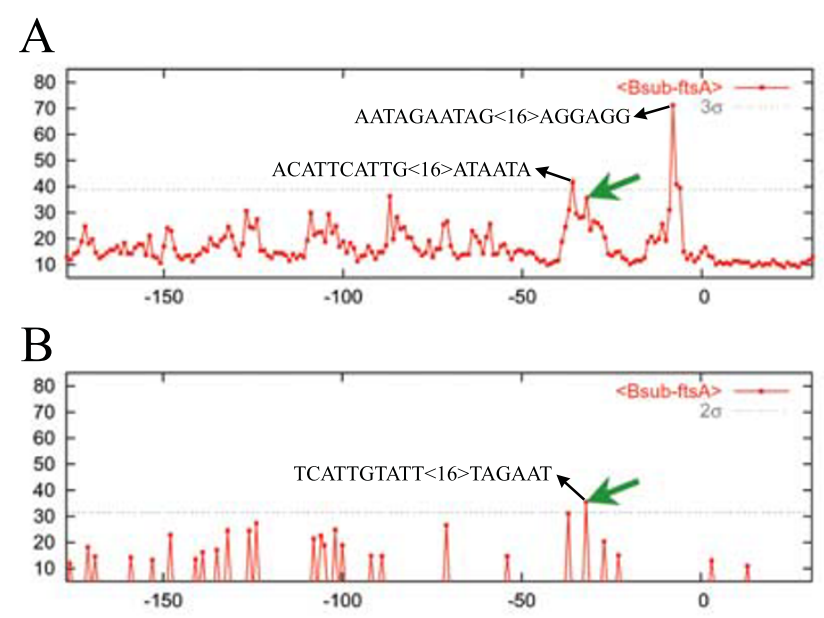

\section{Figure 4}

The application of a simple sequence-dependent filter and the subsequent diminution of the threshold allows the detection of an otherwise missed promoter. Graphs reporting scores calculated for all hexanucleotide pairs located in the enlarged IR upstream of the $B$. subtilis ftsA gene. (A) No filter applied. (B) Sequence-dependent filtering ( 3 mismatches and 2 mismatches allowed respectively in the -35 and -10 boxes relative to the $B$. subtilis principal $\sigma$ factor consensus sequence) followed by the reduction of the region-specific threshold from 3 to 2 standard deviations above of the mean.

between bacterial species. In order to clearly demonstrate the potential of genomic distribution as an indicator of DNA motif function, we have developed an algorithm that can identify a significant fraction of principal $\sigma$ factor-dependent promoters in any prokaryotic organism, using only a genome annotation and a synthetic matrix (i.e. the general matrix, which was obtained from a training set composed of experimentally identified promoters from ten bacterial species). Promoter predictions were also made and experimentally verified, thus highlighting the potential of our approach for promoter identification in various prokaryotic organisms. Overall, our strategy yielded results similar to those from other studies considering an equivalent amount of FP/100 nt (see Additional file 5: Comparison with other bacterial promoter prediction approaches). However, our algorithm took advantage of a yet unexploited concept, can be used in a wide variety of organisms, required almost no previous knowledge of promoter sequences to be effective, and can be combined with other methodologies. The fact that our general matrix allowed the detection of more promoters in all tested genomes relative to the E. coli and B. subtilis principal $\sigma$ factor normalized PSWM (see Additional file 6: Comparison with normalized PSWM scoring function) also supports the idea that genomic distribution of pro- 
Table 2: Experimental validation of predicted transcriptional start sites.

\begin{tabular}{|c|c|c|c|}
\hline Organism & Gene Name & Promoter sequence & Z-Score \\
\hline E. coli & yfgA & GTGGGCTTTGTCACGAGCACACAGACGGTCTIATACTGTATGATAAC & 14.07 \\
\hline E. coli & ygfE & AAAAGGGCTTGTCTCTTCTCATCAGGGTAGCTATAGTGTCGCCCCTT & 9.74 \\
\hline E. coli & secE & ATCATIGCTGAGACAGGCTCTGTTGAGGGCGTATAATCCGAAAAGCT & 7.75 \\
\hline E. coli & $\operatorname{tag}$ & ATATTATTGTCATTGTATGAAGGATATCGGGCATAGTAGCCCTGTAT & 5.90 \\
\hline B. subtilis & proB & AAAAACCTTGACAAGTGTCTTTTTTCTTTGCATAATATAAAAAAATC & 14.20 \\
\hline B. subtilis & $\operatorname{ImrA}$ & AATTTITCTTGACAATTGATGATTGAATCAAGATAATAGACCAGTCA & 11.58 \\
\hline M. tuberculosis & rpsA & GACCGAGTTTGTCCAGCGTGTACCCGTCGAGTAGCCTCGTCAGGTAC & 4.69 \\
\hline
\end{tabular}

Bacterial species, gene names, predicted promoter sequences (underlined - 35 and - 10 boxes), experimentally identified transcriptional start sites (in bold) and the corresponding Z-scores are shown for all tested IRs where a primer extension signal was detected.

moter sequences is easier to transfer across organisms with regards to current promoter sequence models.

Although our approach is based on the genomic distribution of hexanucleotide pairs rather than a direct sequence evaluation, it is still important to know the approximate spacing range that is tolerated by a $\sigma$ factor to efficiently detect the corresponding promoter boxes (data not shown). However, this distance appears to be very similar in most bacteria. The spacing range limitation restrains putative promoter signal contamination by irrelevant hexanucleotide pairs.

An important assumption in our method is that all promoter sequences share a related genomic distribution pattern. However, it is possible that some promoters fall in distinct biological categories or slightly differ between bacterial species. As a consequence, specific matrices could be more adapted to different promoter types. For example, a matrix could be more suitable for promoters containing an extended -10 promoter box. Similarly, very weak promoter sequences could bear an altered distribution pattern when compared to strong promoters.

Another important consideration in our study is the relatively small size of prokaryotic genomes. Since many of these contain only a few million bp, some hexanucleotide pairs are particularly absent from IRs and/or from the whole genome. Therefore, blanks (or 0) in mismatch containing cells can be found in some genome-derived distribution matrices, thus strongly altering the resemblance with the synthetic matrix. Nonetheless, a few promoter sequences containing a blank cell are identified by our approach (see Mtb-rrs in Figures 2C and 3C), although most of them are not (data not shown). Since the score is calculated from the mean of hexanucleotide pairs sharing the same proximal box, a blank occurring only at a particular spacing may not be too detrimental to the overall score of a sequence.
An additional possibility to explain our inability to identify some promoters is that, although present in the genome, some mismatch combinations may be relatively rare, and a small variation in absolute numbers may have a significant impact on $\mathrm{P} / \mathrm{G}$ ratios. In addition, some promoter sequences contain nt triplets corresponding to codons frequently used in translation, which may flatten their distribution bias for IRs. For instance, 19\% of the TP and $44 \%$ of the FN hexanucleotide pairs of E. coli evaluated with the organism-specialized matrix (22\% and $41 \%$ respectively with the general synthetic matrix) include a triplet which is used as a codon more frequently than average. Thus, almost half of the FN in E. coli seem to have an impaired distribution profile because of the inclusion of at least one frequent codon.

In spite of the fact that our algorithm was designed for fully sequenced and annotated genomes, preliminary tests suggest that a genomic distribution calculated from a closely related organism can be used as a reference with similar results (data not shown). Similarly, errors in genomes annotation could theoretically have an impact on the results, albeit we have not observed any significant deterioration of predictions using older versions of the $E$. coli and B. subtilis genome annotations (data not shown).

We have shown that combining different detection strategies by applying a very simple sequence-dependent filter to our promoter predictions significantly decreases the FP rate. Since accuracy is a trade-off between sensitivity and the FP rate, this procedure could allow the reduction of the threshold, thus leading to the detection of more TP and increasing the sensitivity. The integration of a more sophisticated sequence-dependent method to our strategy could be used to further reduce the FP rate. Distance filters were also successfully used by other groups to decrease the number of FPs [24,39]. However, this can hardly be justified in biological terms as underlined by Huerta and Collado-Vides [24]. Moreover, such filters may not be 
suitable for alternative $\sigma$ factor-dependent promoters or other transcription regulators. We have thus decided not to exploit distance constraints, although it remains possible for an eventual user to determine if a putative promoter is located at an appropriate distance from a gene of interest.

\section{Conclusion}

A simple and intuitive concept about the preferential location of regulatory sequences has allowed the identification of principal $\sigma$ factor dependent-promoter sequences in the genome of various bacteria. Minimal information about the structure of the searched pattern was only required for our algorithm to detect these promoters. Moreover, it could be possible to predict promoters in species for which little transcriptional information is available using the proposed general matrix. Since a biased distribution pattern also appear to be conserved for alternative $\sigma$ factors and other regulatory proteins in a variety of prokaryotes, it should be possible to design distribution matrices to identify their corresponding DNA binding sites.

\section{Methods \\ Promoter sequences, corresponding genomes and IRs datasets}

Most of the E. coli and B. subtilis characterized promoter sequence datasets were respectively gathered from EcoCyc version $8.0[41,42]$, and DBTBS release $3.1[43,44]$, and the literature $[5,45]$. To circumvent possible errors in promoter datasets, consistency tests against corresponding genomic sequences were performed [38] with the ASAP gene annotation version m54 for $E$. coli K-12 strain MG1655 [46,47], and SubtiList release R16.1 for B. subtilis $[48,49]$. Binding sites that were not unambiguously detected in their corresponding genome were excluded. The complete corresponding IRs, to which $30 \mathrm{nt}$ were added on both sides, were then extracted from each genome. This resulted in 377 characterized E. coli $\sigma^{70}$ dependent promoter sequences from 335 different enlarged IRs, and 148 B. subtilis $\sigma^{\mathrm{A}}$-dependent promoter sequences from 142 enlarged IRs. The procedure was also applied to promoter sequences found in the MtbRegList database release 1.1 for $M$. tuberculosis [50,51], and for characterized promoters identified from the literature for Corynebacterium glutamicum [52], M. pneumoniae [53], S. coelicolor [54], H. pylori [55-57], C. jejuni [58], B. japonicum [59-64], and S. aureus [65-69] (Table 1). Genome annotations originated from: $M$. tuberculosis H37Rv (TubercuList R6) $[70,71]$, C. glutamicum ATCC13032 (NC_003450.3) [72], M. pneumoniae M129 (NC_000912.1), S. coelicolor A3(2) (NC_003888.3), H. pylori 26695 (PyloriGene R1.6) [73,74], C. jejuni NCTC11168 (NC_002163.1), B. japonicum (NC_004463.1), and S. aureus Mu50 (NC_002758.2). Since there is no principal $\sigma$ factor pro- moter consensus sequence clearly identified for M. tuberculosis, promoter sequences were selected as for groups A and B of Table 1 in Gomez and Smith [75] using the MtbRegList database. Similarly, only S. coelicolor promoter sequences from Table 1 of Strohl [54] were considered. Datasets are available in Additional file 7.

\section{Genome-derived distribution matrices}

Genomic distributions of hexanucleotide pairs were represented by a ratio of the number of hits in IRs located upstream of a gene $(\mathrm{P})$ to total hits in the whole genome (G). Hits were counted only on the functional strand (on the same strand than the following coding sequence) for all spacings inside the allowed spacing range. Identical hexanucleotide pairs with different spacer length will thus have the same genome-derived distribution matrix provided that their respective spacings are included in the allowed range. The genomic distribution of up to three exclusive mismatches per hexanucleotide was also reported in genome distribution matrices. Ratios at various mismatches combinations were reported in genomederived distribution matrices of dimension $4 \times 4$ (Figure 1 , 2 ). Columns and rows respectively represent mismatches in the -10 (proximal) and -35 (distal) boxes.

\section{Synthetic matrices}

248371200 distribution matrices were generated in silico and referred to as "synthetic matrices". To create these, the genome-derived distribution matrices of almost all characterized promoter sequences available were analyzed, and the range of variation in each cell was determined in accordance with the observed ratios. The range and step length was independently established in each cell. Detailed information about synthetic matrices is available in Additional file 2.

\section{Score calculation}

To calculate a score, the genome-derived distribution matrix of a hexanucleotide pair was compared to a synthetic matrix. The analytical approach was inspired by the image processing field and involved four components, each representing the mean of square differences between matrices: $R_{1}$ is calculated on the raw data of the matrices, and $R_{2}$ to $R_{4}$ are respectively calculated on the horizontal, vertical and diagonal directional derivatives of matrices to evaluate the three different slopes of the matrices. Each slope is related to the representation of the genomic distribution of hexanucleotide pairs (proximal and distal boxes). A weight ( $\mathrm{w}$ ) of $1 / 4$ is next applied to each component. The final score $=1 /\left(w R_{1}+w R_{2}+w R_{3}+w R_{4}\right)$. See Additional file 3 for a detailed example of score calculation. 


\section{IR scanning}

IR scanning was accomplished by taking the first six most upstream nt of an enlarged IR along with the downstream hexanucleotide window located at the shortest distance within the specified spacing range. A genome-derived distribution matrix was then generated and a score was calculated with the above described score metric. This procedure was repeated for all allowed spacings by moving the downstream hexanucleotide window by one nt. The upstream hexanucleotide was next moved by one nt and the same procedure was repeated until all appropriate hexanucleotide pairs of the region were processed. The mean of values obtained for all hexanucleotide pairs sharing the same proximal box were then plotted on a graph (Figure 3). Using the maximum values instead of the mean gave very similar results (data not shown). Two thresholds were selected. The region threshold (tR) was set at three standard deviations above the mean of all points from a specific IR. The genome threshold (tG) was set at two standard deviations from the mean of all points from all IR of a genome. $\mathrm{tR}$ and $\mathrm{tG}$ were optimized using E. coli and B. subtilis promoters data (data not shown). The value of any point had to be higher than both thresholds to be considered as a candidate promoter. All adjacent points above thresholds were combined in one peak and represented by their highest point. The widest peak has 6 points and the mean is 1.25 point per peak. A peak had to be located within $4 \mathrm{nt}$ of an experimentally identified TSS to be considered as a TP. All other points above thresholds were considered as FPs. Points representing characterized hexanucleotide pairs below the highest threshold were considered as FNs, while all other points below this threshold were considered as TNs. According to Tompa et al., sensitivity is defined as $\mathrm{TP} /(\mathrm{TP}+\mathrm{FN})$, specificity as $\mathrm{TN} /$ $(\mathrm{TN}+\mathrm{FP})$, precision (or positive predictive value) as $\mathrm{TP} /$ $(\mathrm{TP}+\mathrm{FP})$, and performance as $\mathrm{TP} /(\mathrm{TP}+\mathrm{FN}+\mathrm{FP})[38]$.

\section{Evaluation of synthetic matrices}

The evaluation of over 248 million synthetic matrices on the 625 enlarged IRs (containing 684 characterized promoter sequences from the ten genomes mentioned in Table 1) was performed on the Mammouth Linux cluster of the Université de Sherbrooke (1808 processors, 7.6 Tflops, 5.5 TB of RAM, 160 TB of HD). The performance score for each synthetic matrix was calculated and the best matrix selected as the specific matrix for each genome (Table 1). The general synthetic matrix was selected from the sum of the relative performances of each matrix on each genome following $S=\left(\operatorname{sum}_{i}\left(\operatorname{perf}_{i j} / \operatorname{maxPerf}_{j}\right)\right)$ where perf $_{i j}$ represents the performance score of a given matrix in

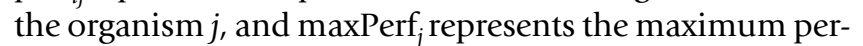
formance score of all matrices in the organism $j$.

\section{Cross-validation tests}

Three-fold cross-validation tests were conducted with $1 \%$ of the synthetic matrices randomly chosen from the previously described set. The E. coli and B. subtilis datasets were randomly divided into three groups, and all possible combinations of two groups were used to select new specialized synthetic matrices. The statistical indicators were next calculated on the remaining group. Results are presented in Additional file 4.

\section{PSWM scoring function}

The scoring function of our method was replaced by a function based on PSWM scores. The rest of the IR scanning procedure remained absolutely identical to the initial design. The promoter datasets of E. coli and B. subtilis were used to construct PSWMs, which were normalized according to the intergenic ATGC content of the tested genome. Results are presented in Additional file 6.

\section{Shuffled genome}

Two shuffled genomes were created. First, the regions (genes and IRs) were independently shuffled to conserve the possible AT bias of IR [40]. The second type was made on the entire genome so that no bias is kept. Shuffled genomes were next used to calculate genome-derived distribution matrices to assess the same enlarged IRs previously analyzed (data not shown).

\section{Codon usage evaluation}

By definition, a hexanucleotide contains 4 overlapping codons. The mean of the utilization ratio of the eight codons of a hexanucleotide pair was thus compared to the average usage frequency of a codon (15.62/1000 residues for $E$. coli) to evaluate if there is a difference between hexanucleotide pair sequences precisely identified (TP) or missed (FN) (data not shown). Codon usages were taken from the Codon Usage Database [76].

\section{Biochemical validation of predicted promoters}

All IRs for which no promoter sequence is characterized in B. subtilis, E. coli and M. tuberculosis, were analyzed with their respective organism-specialized matrix to predict putative promoters. In order to validate some predicted promoters under the control of the housekeeping $\sigma$ factor, predictions were selected on the basis of the putative function of their corresponding gene, the Z-score, the loci organization and the promoter sequences. Validation of the M. tuberculosis prediction was made on the closely related non-pathogenic $M$. bovis BCG-Russia. E. coli $\mathrm{K} 12$ ATCC10798 and B. subtilis NIG2001 [77] were grown in LB medium. M. bovis BCG-Russia was grown in Middlebrook 7H9 medium supplemented with Albumine-Dextrose-Saline, Tween 80 and cycloheximide. All cultures were harvested at an $\mathrm{OD}_{600}$ between 0.6 and 0.8 and RNA was extracted using the Ribopure RNA extraction kit 
(Ambion) or the RNeasy kit (Qiagen). RNA was quantified by spectrophotometry and integrity was verified on formaldehyde denaturing gel. Primer extensions were performed according to standard procedures. Between 30 and $60 \mu \mathrm{g}$ of RNA were used for each reaction. Extension products were migrated on $5 \mathrm{M}$ urea- $6 \%$ acrylamide sequencing gels along with sequencing reactions. IRs were cloned in pCR2.1-TOPO TA cloning vector (Invitrogen) or pdrive TA cloning vector (Qiagen). Oligonucleotide primers are listed in Additional file 8. Sequencing ladders were produced with the Sequenase 2.0 kit (USB) according to the manufacturer's instructions. Gels were scanned using a Molecular Dynamics Storm 840 Phosphorimager.

\section{Authors' contributions}

PEJ contributed to the basic concept of the study, elaborated the design of the study, performed almost all the analysis, and wrote the manuscript. SR contributed to the design of the study, carried out the biochemical validation, and wrote the manuscript. LG and JG contributed to the design and coordination of the study, and helped writing the manuscript. RB elaborated the basic concept of the study, contributed to the design and coordination of the study, and helped writing the manuscript. All authors read and approved the final manuscript.

\section{Additional material}

\section{Additional file 1}

A schematic description of the procedures used in this work. A schematic description of the procedures used in this work.

Click here for file

[http://www.biomedcentral.com/content/supplementary/14712105-7-423-S1.pdf]

\section{Additional file 2}

Detailed information on the generation of synthetic matrices. Minimum and maximum values with step length and number of values for the construction of each cell of the synthetic matrices.

Click here for file

[http://www.biomedcentral.com/content/supplementary/14712105-7-423-S2.pdf]

\section{Additional file 3}

Detailed example of score calculation. Detailed example for each step of score calculation.

Click here for file

[http://www.biomedcentral.com/content/supplementary/1471-

2105-7-423-S3.xls]

\section{Additional file 4}

Three fold cross-validation results. Cross-validation results for each of the "training" and "testing" phases, along with scores between matrices identified by each training phases and their respective organism-specialized matrix.

Click here for file

[http://www.biomedcentral.com/content/supplementary/14712105-7-423-S4.pdf]

\section{Additional file 5}

Comparison with other bacterial promoter prediction approaches. Comparison between our method and other bacterial promoter prediction approaches. Three variations of the region-specific threshold are shown for organism-specialized matrices.

Click here for file

[http://www.biomedcentral.com/content/supplementary/14712105-7-423-S5.pdf]

\section{Additional file 6}

Comparison with normalized PSWM scoring function. Comparison of our integral method with a method in which the scoring function was replaced by normalized PSWM scores.

Click here for file

[http://www.biomedcentral.com/content/supplementary/14712105-7-423-S6.pdf]

\section{Additional file 7}

Datasets of promoter sequences and enlarged IRs. Fasta format where the title line of each sequence contains the following information: > "Organism name" _ "Gene name" "-35 hexanucleotide" < "Spacing" > "-10 hexanucleotide" _ "Name of the sigma factor when available" _ "Exclusive distance between the 3' base of the -10 hexanucleotide and the first base of the start codon" _ "Length of the complete IR". The $30 \mathrm{nt}$ upstream and downstream each IR are also respectively included.

Click here for file

[http://www.biomedcentral.com/content/supplementary/14712105-7-423-S7.txt]

\section{Additional file 8}

Primer sequences used for biochemical validations. Primer sequences used for biochemical validations.

Click here for file

[http://www.biomedcentral.com/content/supplementary/14712105-7-423-S8.pdf]

\section{Acknowledgements}

Authors would like to thank François Deschênes, Alain Gervais, and the team of the Centre de Calcul Scientifique at Université de Sherbrooke for their help with the development of the algorithm. We also thank François Robert, Mathieu Blanchette, Benoît Leblanc and Karine Lemieux for their valuable comments on the manuscript, and Peter Mueller for the B. japonicum dataset. This work was supported by a NSERC-Genomic research grant to RB, LG, and JG. LG holds a Canada Research Chair on mechanisms of gene transcription. JG is a member of the RQCHP, providing an access to the Mammouth Linux cluster of the Université de Sherbrooke. PÉJ and SR are respectively the recipients of a FQRNT and a NSERC Ph.D. scholarship.

\section{References}

I. Gross CA, Chan C, Dombroski A, Gruber T, Sharp M, Tupy J, Young $B$ : The functional and regulatory roles of sigma factors in transcription. Cold Spring Harb Symp Quant Biol 1998, 63:141-55.

2. Gruber TM, Gross CA: Multiple sigma subunits and the partitioning of bacterial transcription space. Annu Rev Microbiol 2003, 57:44I-66.

3. Murakami KS, Masuda S, Campbell EA, Muzzin O, Darst SA: Structural basis of transcription initiation: an RNA polymerase holoenzyme-DNA complex. Science 2002, 296: 1285-90. 
4. Dombroski AJ, Johnson BD, Lonetto M, Gross CA: The sigma subunit of Escherichia coli RNA polymerase senses promoter spacing. Proc Natl Acad Sci U S A 1996, 93:8858-62.

5. Helmann JD: Compilation and analysis of Bacillus subtilis sigma A-dependent promoter sequences: evidence for extended contact between RNA polymerase and upstream promoter DNA. Nucleic Acids Res 1995, 23:235।-60.

6. Mitchell JE, Zheng D, Busby SJ, Minchin SD: Identification and analysis of 'extended - I0' promoters in Escherichia coli. Nucleic Acids Res 2003, 31 : 4689-95.

7. deHaseth PL, Zupancic ML, Record MT jr: RNA polymerase-promoter interactions: the comings and goings of RNA polymerase. J Bacteriol 1998, I80:3019-25.

8. Record MT, Reznikoff WS, Craig ML, McQuade KL, Schlax PJ: Escherichia coli RNA polymerase (Es70), promoters, and the kinetics of the steps of transcription initiation. In Escherichia coli and Salmonella typhimurium: Cellular and molecular biology Volume I. 2nd edition. Edited by: Neidhardt FC, Ingraham JL, Lin ECC, Low KB, B Magasanik W S. RCI Washington, D.C.: ASM Press; 1996:792-820.

9. Lawrence CE, Altschul SF, Boguski MS, Liu JS, Neuwald AF, Wootton JC: Detecting subtle sequence signals: a Gibbs sampling strategy for multiple alignment. Science 1993, 262:208-I4.

10. Bailey TL, Elkan C: Fitting a mixture model by expectation maximization to discover motifs in biopolymers. Proc Int Conf Intell Syst Mol Biol 1994, 2:28-36.

II. Sinha S, Tompa M: A statistical method for finding transcription factor binding sites. Proc Int Conf Intell Syst Mol Biol 2000, 8:344-54.

12. van Helden J, Rios AF, Collado-Vides J: Discovering regulatory elements in non-coding sequences by analysis of spaced dyads. Nucleic Acids Res 2000, 28: 1808-18.

13. Rajewsky N, Socci ND, Zapotocky M, Siggia ED: The evolution of DNA regulatory regions for proteo-gamma bacteria by interspecies comparisons. Genome Res 2002, I 2:298-308.

14. McGuire AM, Hughes JD, Church GM: Conservation of DNA regulatory motifs and discovery of new motifs in microbial genomes. Genome Res 2000, I0:744-57.

15. McCue L, Thompson W, Carmack C, Ryan MP, Liu JS, Derbyshire V, Lawrence CE: Phylogenetic footprinting of transcription factor binding sites in proteobacterial genomes. Nucleic Acids Res 200I, 29:774-82.

16. Gelfand MS, Koonin EV, Mironov AA: Prediction of transcription regulatory sites in Archaea by a comparative genomic approach. Nucleic Acids Res 2000, 28:695-705.

17. Blanchette M, Schwikowski B, Tompa M: An exact algorithm to identify motifs in orthologous sequences from multiple species. Proc Int Conf Intell Syst Mol Biol 2000, 8:37-45.

18. Kanhere A, Bansal M: A novel method for prokaryotic promoter prediction based on DNA stability. BMC Bioinformatics 2005, 6:1-10

19. Galas DJ, Eggert M, Waterman MS: Rigorous pattern-recognition methods for DNA sequences. Analysis of promoter sequences from Escherichia coli. I Mol Biol 1985, I 86: I 17-28.

20. Stormo GD: DNA binding sites: representation and discovery. Bioinformatics 2000, 16:16-23.

21. Stormo GD, Schneider TD, Gold L, Ehrenfeucht A: Use of the 'Perceptron' algorithm to distinguish translational initiation sites in E. coli. Nucleic Acids Res 1982, 10:2997-301 I.

22. Munch R, Hiller K, Grote A, Scheer M, Klein J, Schobert M, Jahn D: Virtual Footprint and PRODORIC: an integrative framework for regulon prediction in prokaryotes. Bioinformatics 2005, $21: 4187-9$.

23. Robison K, McGuire AM, Church GM: A comprehensive library of DNA-binding site matrices for $\mathbf{5 5}$ proteins applied to the complete Escherichia coli K-I2 genome. J Mol Biol 1998, 284:24I-54.

24. Huerta AM, Collado-Vides J: Sigma70 promoters in Escherichia coli: specific transcription in dense regions of overlapping promoter-like signals. J Mol Biol 2003, 333:261-78.

25. Horton PB, Kanehisa M: An assessment of neural network and statistical approaches for prediction of $E$. coli promoter sites. Nucleic Acids Res 1992, 20:4331-8.

26. Jarmer H, Larsen TS, Krogh A, Saxild HH, Brunak S, Knudsen S: Sigma $A$ recognition sites in the Bacillus subtilis genome. Microbiology 2001, I 47:2417-24
27. Petersen L, Larsen TS, Ussery DW, On SL, Krogh A: RpoD promoters in Campylobacter jejuni exhibit a strong periodic signal instead of a $\mathbf{- 3 5}$ box. J Mol Biol 2003, 326:136I-72.

28. Gordon JJ, Towsey MW, Hogan JM, Mathews SA, Timms P. Improved prediction of bacterial transcription start sites. Bioinformatics 2006, 22:|42-8.

29. Vanet A, Marsan L, Labigne A, Sagot MF: Inferring regulatory elements from a whole genome. An analysis of Helicobacter pylori sigma(80) family of promoter signals. J Mol Biol 2000 , 297:335-53

30. Eskin E, Keich U, Gelfand MS, Pevzner PA: Genome-wide analysis of bacterial promoter regions. Pac Symp Biocomput 2003:29-40.

31. Mwangi MM, Siggia ED: Genome wide identification of regulatory motifs in Bacillus subtilis. BMC Bioinformatics 2003, 4:18.

32. Li H, Rhodius V, Gross C, Siggia ED: Identification of the binding sites of regulatory proteins in bacterial genomes. Proc Natl Acad Sci U S A 2002, 99: I I 772-7.

33. Studholme DJ, Bentley SD, Kormanec J: Bioinformatic identification of novel regulatory DNA sequence motifs in Streptomyces coelicolor. BMC Microbiol 2004, 4:14.

34. Smith I, Bishai WR, Nagaraja V: Control of Mycobacterial Transcription. In Tuberculosis and the Tubercule Bacillus Volume I. I st edition. Edited by: Cole ST, McMurray DN, Eisenach K, Gicquel B, Jacobs WR. Washington, D.C.: ASM Press; 2005:219-3I.

35. Young BA, Gruber TM, Gross CA: Views of transcription initiation. Cell 2002, I 09:4I7-20.

36. Browning DF, Busby SJ: The regulation of bacterial transcription initiation. Nat Rev Microbiol 2004, 2:57-65.

37. Fenton MS, Gralla JD: Function of the bacterial TATAAT -10 element as single-stranded DNA during RNA polymerase isomerization. Proc Natl Acad Sci U S A 200 I, 98:9020-5.

38. Tompa M, Li N, Bailey TL, Church GM, De Moor B, Eskin E, Favorov $\mathrm{AV}$, Frith $\mathrm{MC}$, Fu $\mathrm{Y}$, Kent WJ, et al:: Assessing computational tools for the discovery of transcription factor binding sites. Nat Biotechnol 2005, 23: I 37-44.

39. Burden S, Lin YX, Zhang R: Improving promoter prediction for the NNPP2.2 algorithm: a case study using E-Coli DNA sequences. Bioinformatics 2004.

40. Bentley SD, Parkhill J: Comparative genomic structure of prokaryotes. Annu Rev Genet 2004, 38:771-92.

41. The EcoCyc database [http://ecocyc.org/]

42. Keseler IM, Collado-Vides J, Gama-Castro S, Ingraham J, Paley S Paulsen IT, Peralta-Gil M, Karp PD: EcoCyc: a comprehensive database resource for Escherichia coli. Nucleic Acids Res 2005 , 33(Database):D334-7.

43. The DBTBS database [http://dbtbs.hgc.jp/]

44. Makita Y, Nakao M, Ogasawara N, Nakai K: DBTBS: database of transcriptional regulation in Bacillus subtilis and its contribution to comparative genomics. Nucleic Acids Res 2004, 32(Database):D75-7.

45. Granston AE, Thompson DL, Friedman DI: Identification of a second promoter for the metY-nusA-infB operon of Escherichia coli. I Bacteriol 1990, I 72:2336-42.

46. The ASAP database [https://asap.ahabs.wisc.edu/annotation/php/ home.php]

47. Glasner JD, Liss $P$, Plunkett $G$, Darling A, Prasad $T$, Rusch M, Byrnes A, Gilson M, Biehl B, Blattner FR, et al.: ASAP, a systematic annotation package for community analysis of genomes. Nucleic Acids Res 2003, 3 1:|47-5|.

48. The SubtiList database [http://genolist.pasteur.fr/SubtiList/]

49. Moszer I, Jones LM, Moreira S, Fabry C, Danchin A: SubtiList: the reference database for the Bacillus subtilis genome. Nucleic Acids Res 2002, 30:62-5.

50. The MtbRegList database [http://www.USherbrooke.ca/vers/ MtbRegList]

5I. Jacques PE, Gervais AL, Cantin M, Lucier JF, Dallaire G, Drouin G, Gaudreau L, Goulet J, Brzezinski R: MtbRegList, a database dedicated to the analysis of transcriptional regulation in Mycobacterium tuberculosis. Bioinformatics 2005, 21 l:2563-5.

52. Patek M, Nesvera J, Guyonvarch A, Reyes O, Leblon G: Promoters of Corynebacterium glutamicum. J Biotechnol 2003, 1 04:3 I I-23.

53. Weiner J, Herrmann R, Browning GF: Transcription in Mycoplasma pneumoniae. Nucleic Acids Res 2000, 28:4488-96.

54. Strohl WR: Compilation and analysis of DNA sequences associated with apparent streptomycete promoters. Nucleic Acids Res 1992, 20:961-74. 
55. Forsyth $\mathrm{MH}$, Cover TL: Mutational analysis of the vacA promoter provides insight into gene transcription in Helicobacter pylori. J Bacteriol 1999, I81:2261-6.

56. McGowan CC, Necheva AS, Forsyth MH, Cover TL, Blaser MJ: Promoter analysis of Helicobacter pylori genes with enhanced expression at low pH. Mol Microbiol 2003, 48: 1225-39.

57. Lundström AM, Bölin I: A $\mathbf{2 6} \mathbf{~ k D a}$ protein of helicobacter pylori shows alkyl hydroperoxide reductase (AhpC) activity and the mono-cistronic transcription of the gene is affected by pH. Microb Pathog 2000, 29:257-66.

58. Wösten MM, Boeve M, Koot MG, van Nuene AC, van der Zeijst BA: Identification of Campylobacter jejuni promoter sequences. J Bacteriol 1998, 180:594-9.

59. Beck C, Marty R, Klausli S, Hennecke H, Gottfert M: Dissection of the transcription machinery for housekeeping genes of Bradyrhizobium japonicum. J Bacteriol 1997, 179:364-9.

60. Hamza I, Qi Z, King ND, O'Brian MR: Fur-independent regulation of iron metabolism by Irr in Bradyrhizobium japonicum. Microbiology 2000, 146(pt 3):669-76.

61. Westenberg DJ, Guerinot ML: Succinate dehydrogenase (Sdh) from Bradyrhizobium japonicum is closely related to mitochondrial Sdh. J Bacteriol 1999, I 81 :4676-9.

62. Loh J, Stacey MG, Sadowsky MJ, Stacey G: The Bradyrhizobium japonicum nolA gene encodes three functionally distinct proteins. J Bacteriol I999, I 8 I: I 544-54.

63. LeVier K, Guerinot ML: The Bradyrhizobium japonicum fegA gene encodes an iron-regulated outer membrane protein with similarity to hydroxamate-type siderophore receptors. J Bacteriol 1996, I 78:7265-75.

64. Wang SP, Stacey G: Studies of the Bradyrhizobium japonicum nodD I promoter: a repeated structure for the nod box. J Bacteriol 199I, 173:3356-65.

65. Deora R, Tseng T, Misra TK: Alternative transcription factor sigmaSB of Staphylococcus aureus: characterization and role in transcription of the global regulatory locus sar. J Bacteriol 1997, 179:6355-9.

66. Deora R, Misra TK: Characterization of the primary sigma factor of Staphylococcus aureus. J Biol Chem 1996, 271:21828-34.

67. Manna AC, Bayer MG, Cheung AL: Transcriptional analysis of different promoters in the sar locus in Staphylococcus aureus. J Bacteriol 1998, 180:3828-36.

68. Tegmark K, Morfeldt E, Arvidson S: Regulation of agr-dependent virulence genes in Staphylococcus aureus by RNAIII from coagulase-negative staphylococci. J Bacteriol 1998, 180:3181-6.

69. Tegmark K, Karlsson A, Arvidson S: Identification and characterization of SarHI, a new global regulator of virulence gene expression in Staphylococcus aureus. Mol Microbiol 2000, 37:398-409.

70. The TubercuList database [http://genolist.pasteur.fr/Tubercu List/]

7I. Camus JC, Pryor MJ, Medigue C, Cole ST: Re-annotation of the genome sequence of Mycobacterium tuberculosis H37Rv. Microbiology 2002, I 48:2967-73.

72. The NCBI prokaryote genome annotation [ftp:// ftp.ncbi.nih.gov/genomes/Bacteria/]

73. The PyloriGene database [http://genolist.pasteur.fr/PyloriGene/]

74. Boneca IG, de Reuse H, Epinat JC, Pupin M, Labigne A, Moszer I: A revised annotation and comparative analysis of Helicobacter pylori genomes. Nucleic Acids Res 2003, 3 I: I 704- I 4.

75. Gomez M, Smith I: Determinants of Mycobacterial Gene Expression. In Molecular Genetics of Mycobacteria Volume I. Ist edition. Edited by: Hatfull GF, Jacobs WR. Washington, D.C.: ASM Press; 2000: III-29.

76. The Codon Usage Database [http://www.kazusa.or.jp/codon/]

77. Fujita M, Sadaie Y: Rapid isolation of RNA polymerase from sporulating cells of Bacillus subtilis. Gene 1998, 221 : 185-90.

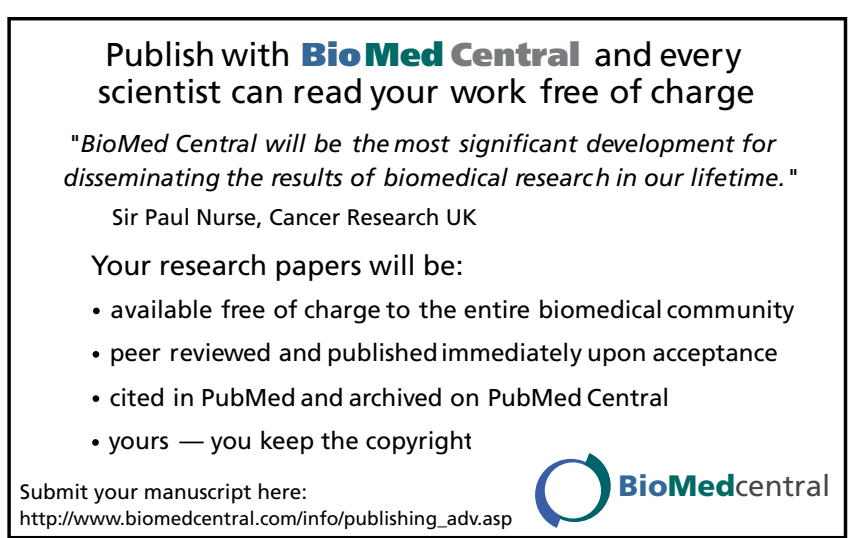

